\title{
Performance Evaluation of Diversity Techniques in IDMA Scheme for Next Generation (4G) in Underwater Wireless Communication
}

\author{
Prachi Tripathi',2, Manoj Kumar Shukla1,2 \\ ${ }^{1}$ Electronics and Communication Engineering Division, Harcourt Butler Technology Institute, Kanpur, India \\ ${ }^{2}$ Gautam Budha Technical University, Lucknow, India \\ Email: dhoolika77@gmail.com
}

Received 29 May 2014; revised 30 June 2014; accepted 11 July 2014

Copyright (C 2014 by authors and Scientific Research Publishing Inc.

This work is licensed under the Creative Commons Attribution International License (CC BY).

http://creativecommons.org/licenses/by/4.0/

\section{(c) (i) Open Access}

\begin{abstract}
This paper provides a review on the diversity techniques of IDMA (Interleave Division Multiple Access) technology in underwater wireless IDMA employs interleavers as the only means in order to distinguish the users. This paper provides a comprehensive study of diversity techniques in IDMA scheme to mitigate the fading issue. In this paper, we compare the different generation diversity techniques in IDMA scheme on computational complexity, bit error rate and memory requirement. Recent advancement in underwater communication is modulation techniques, multiplexing techniques and multiple access techniques. Underwater communication channel is characterized.
\end{abstract}

\section{Keywords}

Wireless Communication, Acoustic Communication, Underwater Communication, Modulation Technique, Multiple Access Scheme, IDMA, CDMA, Interleavers, Underwater Communication Channel

\section{Introduction}

The past few years, the demand of wireless communication services has been grown steadily. The request for bandwidth has started to surpass the availability in wireless networks. Different techniques have been studied to improve the bandwidth, efficiency and increase the number of users within each cell. Cellular radio was origi-

How to cite this paper: Tripathi, P. and Shukla, M.K. (2014) Performance Evaluation of Diversity Techniques in IDMA Scheme for Next Generation (4G) in Underwater Wireless Communication. Wireless Engineering and Technology, 5, 88-98. http://dx.doi.org/10.4236/wet.2014.53010 
nally developed for offering phone services to mobile subscribers. The wireless cellular communication systems with first-generation $(1 \mathrm{G})$ of analogue stage used frequency division multiple access (FDMA) scheme. Due to rapid advances in technologies it has led to the second-generation (2G) of digital stage with time division multiple access (TDMA) and code division multiple access (CDMA) schemes, and now it has stepped into the thirdgeneration (3G). High data rate transmission and burst data traffic which would be the dominant portion of traffic load resolved in 4G generation with IDMA Scheme. The next generation mobile communication systems, i.e. fourth-generation (4G) is required (you can refer to IMT-advanced requirements) to support multiple services in different types of environments. 4G is being developed to facilitate the QoS (quality of service) and required data rate such as wireless broadband access, multimedia messaging service (MMS), video chat, and mobile TV. This paper also focuses the light on various multiple access techniques proposed in 4G communication systems.

Nowadays, the techniques related to future wireless communication have become hot topics for research all over the world. The 3G and beyond systems have been developed to serve people's daily work and life, and to satisfy their demand. Communication generation graph is shown in Figure 1.

\section{Multiple Access Schemes in 4G}

Figure 2 shows the evolution of wireless communication in daily life. 4G communication, data rates up to 100 Mbps for high mobility and up to 1 Gbps for low mobility or local wireless are predicted. Systems fulfilling these requirements are usually considered as fourth-generation (4G) systems. But 3G systems provide data rate of around 3.6 - 7.2 Mbps. Existing multiple access techniques used in 1G/2G/3G systems (such as FDMA/ TDMA/CDMA respectively) are basically suitable for voice communication only and unsuitable for high data rate transmission and burst data traffic which would be the dominant portion of traffic load in 4G system [1] [2]. Multiple access is a data transmission technology that allow a number of users to access a single radio frequency

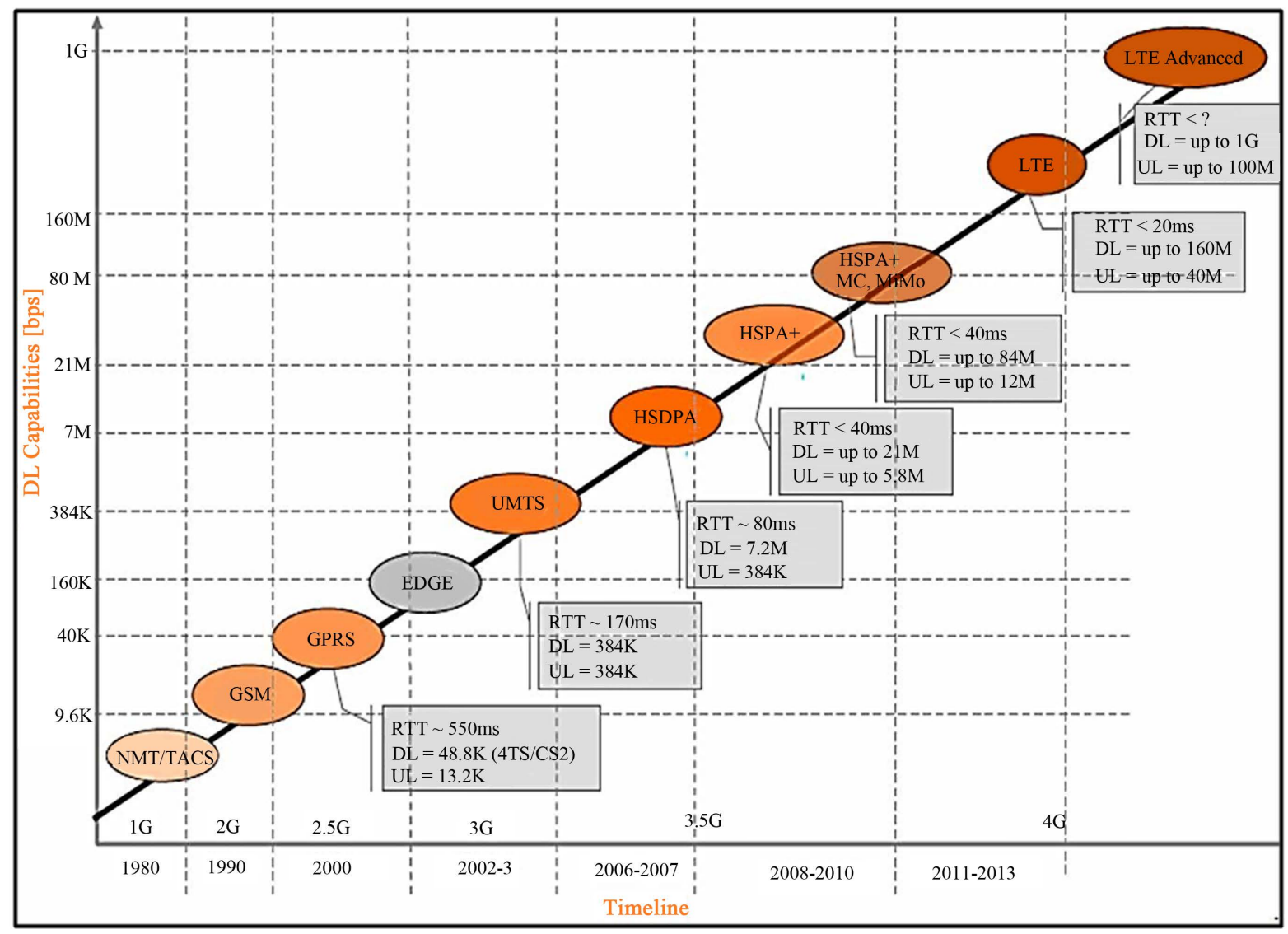

Figure 1. Generation graph. 


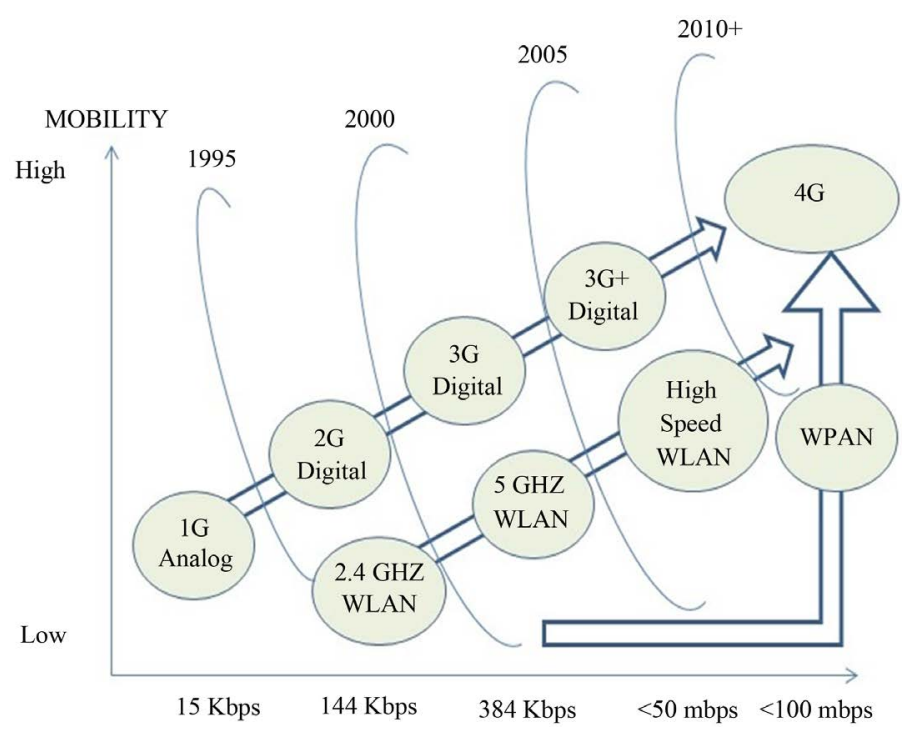

Figure 2. Evolution of wireless communication.

channel without interference [3]. Multiple access technique is one of the key techniques in the wireless communication system [4]. In the past few years, the request for bandwidth has started to surpass the availability in wireless networks. Different techniques have been studied to improve the bandwidth, efficiency and increase the number of users that can be accommodated within each cell. Data rates up to $100 \mathrm{Mbps}$ for high mobility and up to 1 Gbps for low mobility or local wireless are predicted [5].

\subsection{Multiple Access-IDMA Scheme}

IDMA scheme distinguishes users by chip level interleaving method. IDMA scheme can achieve near single user performance in situations with very large numbers of users. Since conventional CDMA systems is limited by multiple access interference (MAI), as well as inter symbol interference (ISI) so IDMA scheme is emerging as the strongest candidate for the third-generation wireless personal communication systems [6] [7].

\subsubsection{Interleaving Principle}

Let $C=\left[c_{0}, c_{1} \ldots c_{N-1}\right]$ be a sequence of length $N$. An interleaver maps $C$ onto a sequence such that $X$ is a permutation of the elements of $\mathrm{C}$. Considering $\mathrm{C}$ and $\mathrm{X}$ as a pair of $\mathrm{N}$-dimensional vectors, there is one-to-one correspondence between each element of $\mathrm{C}$ and each element of $\mathrm{X}$, as shown in Figure 3.

\subsubsection{IDMA Scheme Transmitter and Receiver Structure Structures shown in Figure 4 and Figure 5.}

\subsection{Why IDMA Better than CDMA Schemes in Underwater Communication?}

Figure 6 shows the difference between CDMA and IDMA schemes. With the existing CDMA, high data rates can be achieved by reducing spreading factor or adopting multi-code CDMA, but the former leads to reduced spreading gain against fading and interference, and the latter needs to overcome the interference among spreading sequences. In contrast, high data rate transmission can be achieved in IDMA systems by assigning the FEC codes with high coding rates.

Neglecting intra-cell interference at low computational cost the multiple access interference (MAI) is a major concern for both CDMA and IDMA cellular networks [8]. The existing CDMA mitigates the MAI by multi-user detection (MUD). However, the high computational Cost involved in MUD which limits the high number of user-application in practical systems. In contrast to CDMA, IDMA uses the iterative chip-by-chip (CBC) detection algorithm to combat intra-cell interferences.

The per-user computational complexity of the CBC is independent of the number of users involved. This means that given the same sum-rate, the more users in a system, the less average transmitted sum-power is 

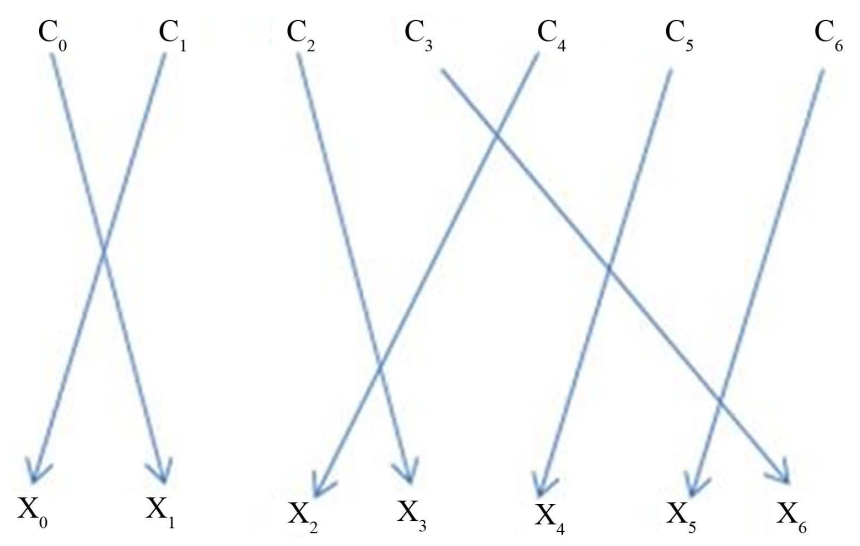

Figure 3. Mechanism of data interleaving.

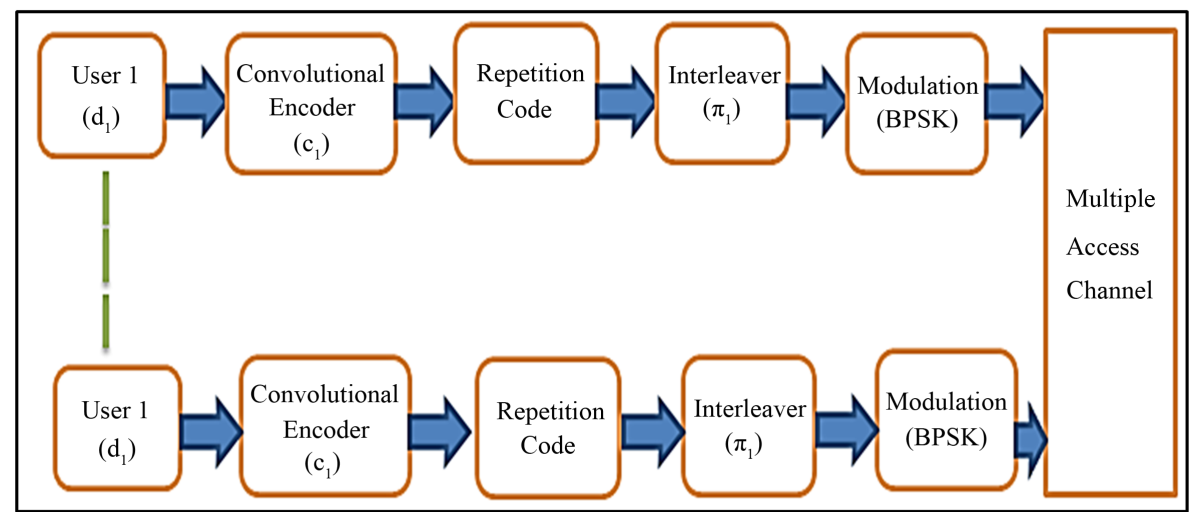

Figure 4. IDMA transmitter mechanism.

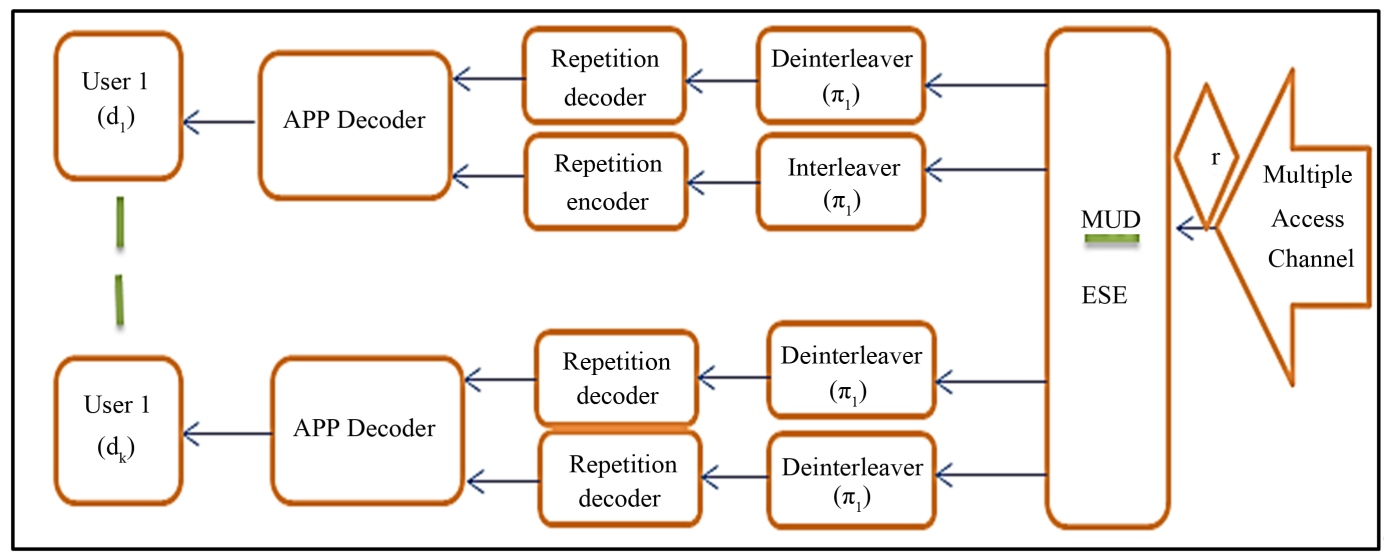

Figure 5. IDMA receiver mechanism.

required. IDMA involves dynamic power control to improve link capacity and guarantee QoS for users.

So IDMA can perform better for large number of users. It supports asynchronous transmission. The orthogonal MA technologies, such as time-division multiple-access (TDMA), frequency-division multiple-access (FDMA) and orthogonal-FDMA (OFDMA), require frame synchronization to maintain orthogonality. In IDMA networks, there is no sophisticated synchronization requirement on data-transmission. Cell specific interleaving brings more robust performance than cell specific scrambling. The advantages of interleaving over scrambling seems very important for cell edge subscriber stations to receive broad cast services such as common signaling broadcasting because some advanced transmitting techniques for unicasting cannot be used for broadcasting [9]. 


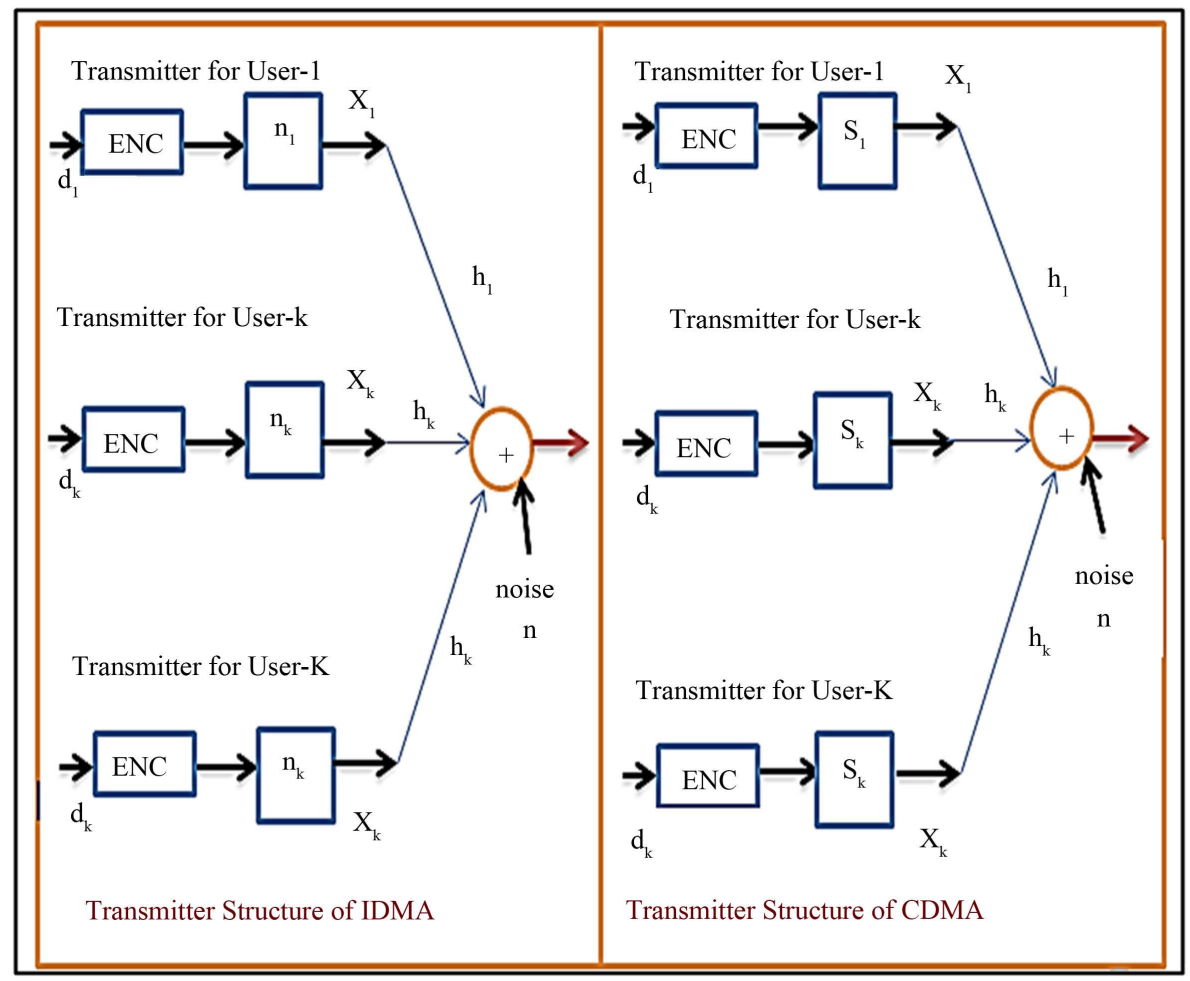

Figure 6. Comparison for IDMA vs CDMA.

\section{Diversity Techniques for 4G IDMA Schemes}

\subsection{Receiver Diversity Analysis for IDMA Scheme}

The block diagram of maximal ratio combining (MRC) diversity with IDMA scheme is shown in Figure 7. In this method, the diversity branches are weighted for maximum SNR. As shown in block diagram in Figure 7, $d_{k}$ is data of $k$ th user, after encoding and spreading the data is randomly interleaved and termed as "chips". Now this chip Signal $k_{x}$ is sent from the transmit antenna, which will propagate from both the channel. If we consider 1 transmit and 2 receive antenna, then channel between transmit antenna and the first received antenna is $h_{0}$ and between the transmit antenna and second receive antenna one is denoted by $h_{1}$. The channel can be modeled having magnitude and phase response. So,

$$
\begin{aligned}
& h_{0}=\alpha_{0} \mathrm{e}^{i \theta_{0}} \\
& h_{1}=\alpha_{1} \mathrm{e}^{i \theta_{1}}
\end{aligned}
$$

Noise can be added at both the receiver. The resulting received signals are

$$
\begin{aligned}
R_{0} & =h_{0} x_{k}+n_{0} \\
R_{1} & =h_{1} x_{k}+n_{1}
\end{aligned}
$$

where, $n_{0}$ and $n_{1}$ represents the noise and interference at both the receiver separately. Now the Receiver combining scheme for two branches MRRC can be written as

$$
\overline{X_{k}}=h_{0} * R_{0}+h_{1} * R_{1}
$$

Now this output of maximal ratio combiner can fed to the detector for the proper estimation of transmitted signal $x_{k}$.

\subsection{Transmit Diversity Analysis for IDMA Scheme}

In this section transmit diversity is discussed with IDMA communication system; Figure 8 is showing the 


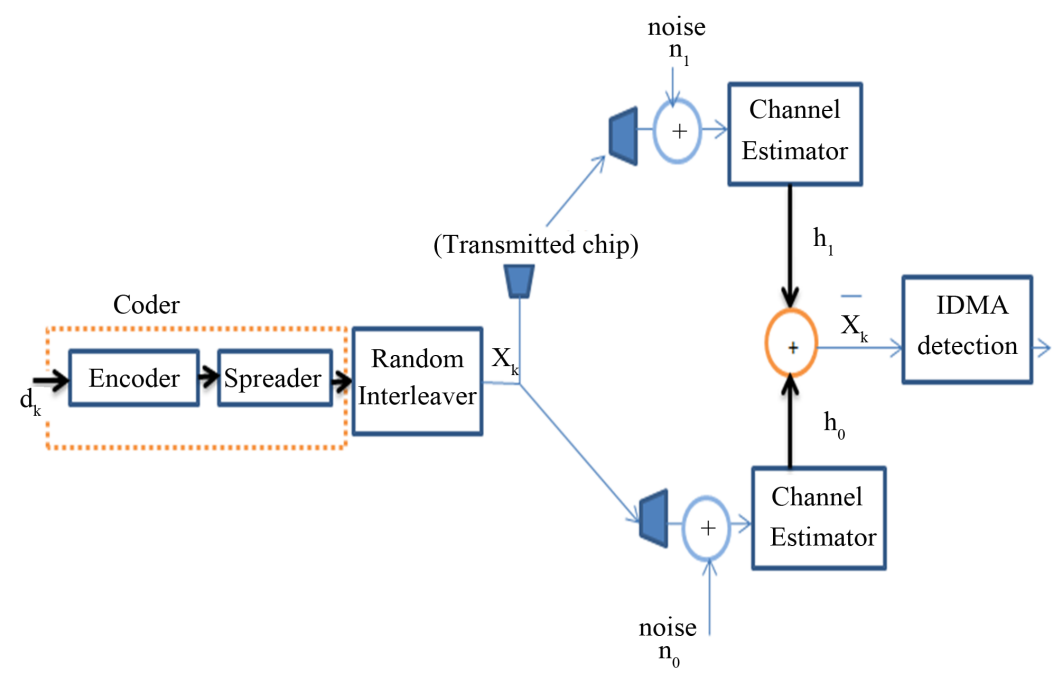

Figure 7. IDMA Scheme with proposed two branches MRRC diversity scheme for $k$ th user.

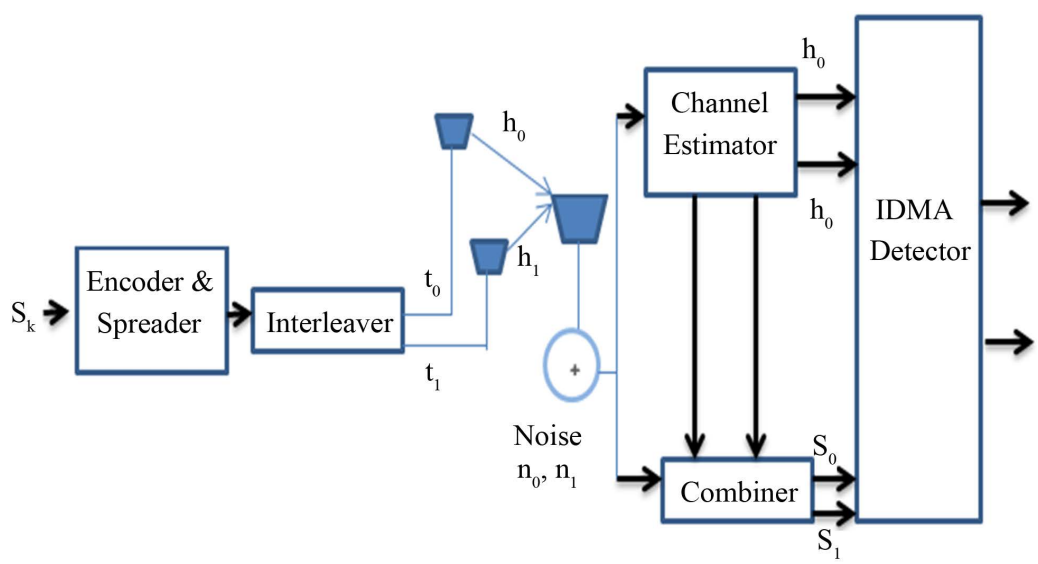

Figure 8. IDMA scheme with proposed transmitter diversity scheme for $k$ th user.

arrangements for $k$ th user. After encoding and spreading the data is interleaved then sent by two transmit antenna.

If two transmit antenna and one receive antenna system is used, then channel between first transmit antenna $\left(t_{0}\right)$ and receiver antenna is $h_{0}$ and between second transmit antenna $\left(t_{1}\right)$ is $h_{1}$. So, the channel can be modeled like:

$$
\begin{gathered}
h_{0}=\alpha_{0} \exp \left(j \theta_{0}\right) \\
h_{1}=\alpha_{1} \exp \left(j \theta_{1}\right)
\end{gathered}
$$

At a given symbol period two signals are simultaneously transmitted from two antennas. The signal transmitted from antenna zero is $S_{0}$ and antenna one is $S_{1}$. During next symbol period conjugate of $\left(-S_{1}\right)$ is transmitted from antenna zero and conjugate signal $S_{0}$ is transmitted from antenna one, i.e. transmitted symbols are space time encoded. Now the received signal can be written as

$$
\begin{aligned}
& r_{0}=r(t)=h_{0} s_{0}+h_{1} s_{1}+n_{0} \\
& r_{1}=r(t+T)=h_{0} s_{1}^{*}+h_{1} s_{0}^{*}+n_{1}
\end{aligned}
$$

These two signals will be received by the same antenna after a delay of $T$ (symbol period).

$$
\widetilde{s_{0}}=h_{0}^{*} r_{0}+h_{1} r_{1}^{*}
$$




$$
\widetilde{s_{1}}=h_{1}^{*} r_{0}-h_{0} r_{1}^{*}
$$

Solving Equations (7)-(9), we can write

$$
\begin{aligned}
& \widetilde{s_{0}}=\left(\alpha_{0}^{2}+\alpha_{1}^{2}\right) s_{0}+n_{0} h_{0}^{*}+h_{1} n_{1}^{*} \\
& \widetilde{s_{1}}=\left(\alpha_{0}^{2}+\alpha_{1}^{2}\right) s_{1}+h_{0} n_{1}^{*}+n_{0} h_{1}^{*}
\end{aligned}
$$

Now finally these two signals fed in to the CBC detection based IDMA detector.

\section{Performance Evaluation}

Performance of diversity techniques can be check on random interleaver (RI) and tree based interleaver (TBI) for 4G spectrum bandwidth for 15 iterations, as shown in Figure 9.

\subsection{Performance of RI-IDMA with Transmitter and Receiver Diversity}

Figure 10 demonstrates the performance of IDMA scheme with using random interleaver. Here in receiver as well as in transmit diversity two branches maximal ratio combining scheme is used for implementation of space diversity technique. In this case, the degree of complexity remains similar to that in simple IDMA systems. The BER performance with maximal ratio combining diversity is better than without using any diversity technique in fading environment.

\subsection{Performance of TBI-IDMA with Transmitter and Receiver Diversity}

Figure 11 shows the BER performance of IDMA scheme using tree based interleaver with both diversity schemes. From this figure we can see that the performance of IDMA system with MRC diversity is far better

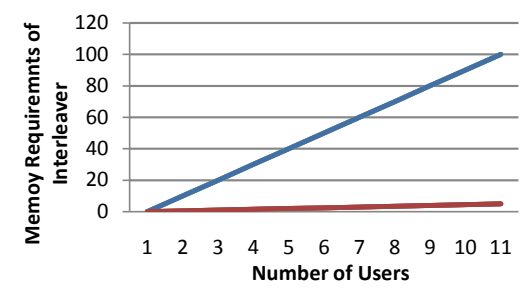

Figure 9. Comparison of memory requirement random interleaver and tree based interleaver.

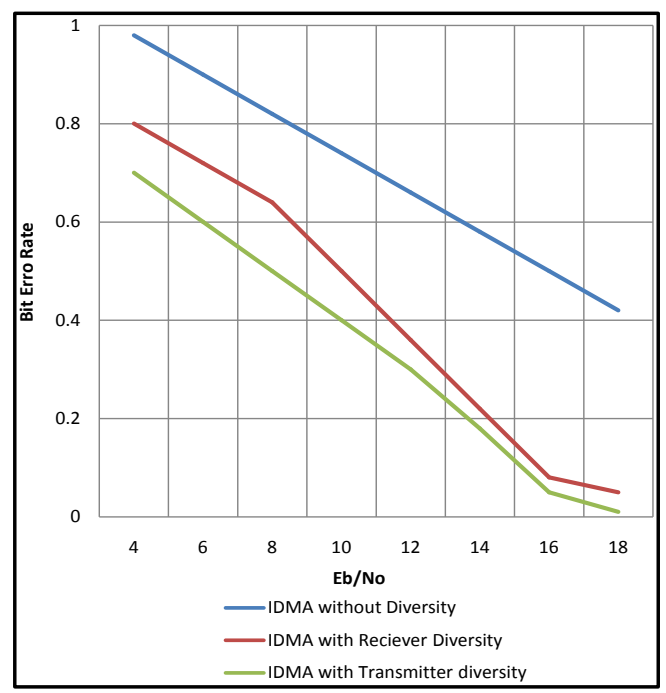

Figure 10. Performance of RI-IDMA with transmitter and receiver diversity. 


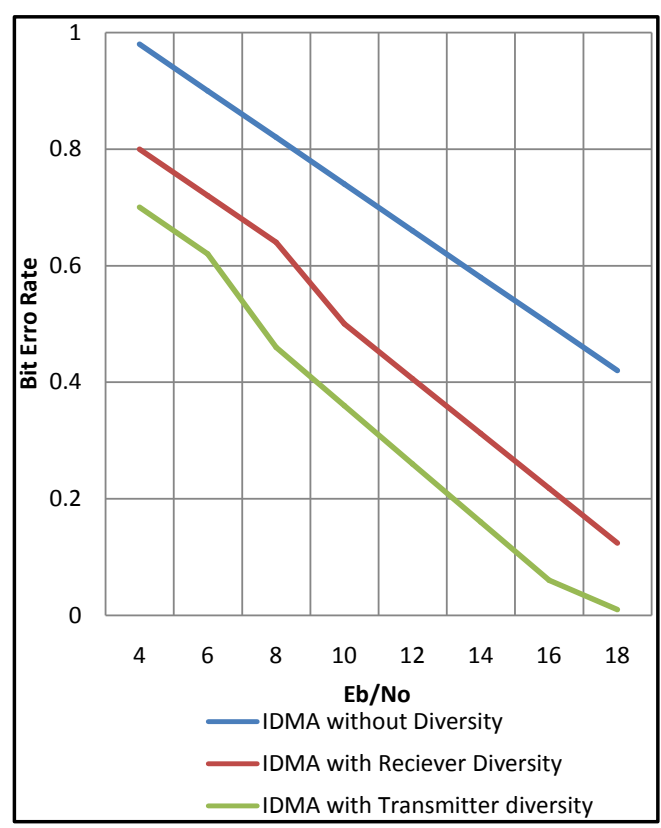

Figure 11. Performance of TBI-IDMA with transmitter and receiver diversity.

than that without diversity also the performance of Tree based interleaver comes out to better in the case of transmit diversity.

\section{Underwater Communication}

Underwater communication technique is used for sending and receiving message below water. There are several ways of employing such communication but the most commonly used are hydrophones. Under water communication is difficult than ground communication due to several factors including time, variations in the channel, multi-path propagation, small available bandwidth and strong signal attenuation, especially over long distances. In underwater communication date rates are low as compared to other communication, thus underwater communication uses acoustic waves instead of electromagnetic waves. Hence it is also called as underwater acoustic communication [10] [11].

Several systems are designed and manufactured commercially and mostly based on analogue technology and employing Single Side Band (SSB) modulation. Technically all these systems are behind the advanced digital technology of mobile radio communication systems. Apart from the modulation scheme indulged, analogue systems have other inherent shortcomings; for example; private communications between channels are not easily achieved and multipath propagation can degrade the quality of the transmitted data. These limitations of analog communication can be overcome by only implementing digital technology as it is now common for mobile telephones. This is the reason by which several experimental systems for digital underwater voice communications have been developed recently.

Since underwater wireless communication channel has limited bandwidth, transmission of quantized speech samples at high bit rates is also restricted, hence speech signals need to be compressed. There are some internationally recommended low bit rate speech coding techniques available at transmission rates of $2.4 \mathrm{kbit} / \mathrm{s}$ to 16 $\mathrm{kbit} / \mathrm{s}$ [12].

In wireless communication channel it is often desirable to allow the subscriber to send the information to the base station while receiving the information from the base station. There are different ways to allow access through the channel. This includes mainly the following:

1) Frequency Division Multiple-Access (FDMA).

2) Time Division Multiple-Access (TDMA).

3) Code Division Multiple-Access (CDMA).

4) Space Division Multiple-Access (SDMA). 
5) Interleave Division Multiple-Access (IDMA).

FDMA, TDMA and CDMA are mostly recommended multiple access techniques that are used to share the available bandwidth in a wireless communication system. Depend upon how the available bandwidth is allocated and used by the users these techniques can be classified as narrowband systems and wideband systems.

\section{Underwater Communication Channel}

The three distinguishing features of this channel are frequency-dependent propagation loss, multipath, and low speed-sound propagation. None of these characteristics are nearly as applied in land-based radio channels, the fact that makes underwater wireless communication extremely difficult, and necessitates dedicated to system design [13] [14].

AWGN: In order to simulate noise in the channel, additive white Gaussian noise (AWGN) added to the transmitted signal. The feature of this noise is uncorrelated and has zero mean. This is only done by simply adding the noise factor to the transmitted signal. The variance of the noise defines the level of the noise and it is calculated from a given signal to the noise ratio.

Phase Error: During the data transmission time phase error can occur. This is the reason it is important to either use carrier tracking at the receiver or differential coding.

Multipath: As shown in Figure 12, in underwater communications multipath inflicts serious distortions to the transmitted and received signal. This is due to long channel impulse response caused by the low wave speed in the water.

Synchronization Error: When a signal transmit or travel via a complicated communication channel it can change drastically. This change can lead to an error in the synchronization at the receiver end.

\section{Other Techniques in Underwater Communication}

\subsection{Hydrophones}

It is a microphone designed to be used underwater for recording or listening the underwater communication. Hydrophones are based on piezoelectric transuder that generates electricity when subjected to a pressure change. Converts acoustic waves into electrical energy, It Cannot measure multiple scalar quantities, and has Single Input Single.

\subsection{Sonar}

Sonar originally pronounced for sound navigation and ranging. It is a technique that uses sound propagation to navigate, communicate with or detect other vessels. Two type of technology in sonar; active sonar is emitting pulses of sound and listening for echoes, passive sonar is essentially listening the sound made by vessels.

\section{Securing Underwater Network}

\subsection{Attacks on Underwater Networks}

Underwater communication network suffers from the vulnerabilities. High data bit error rates cause packet

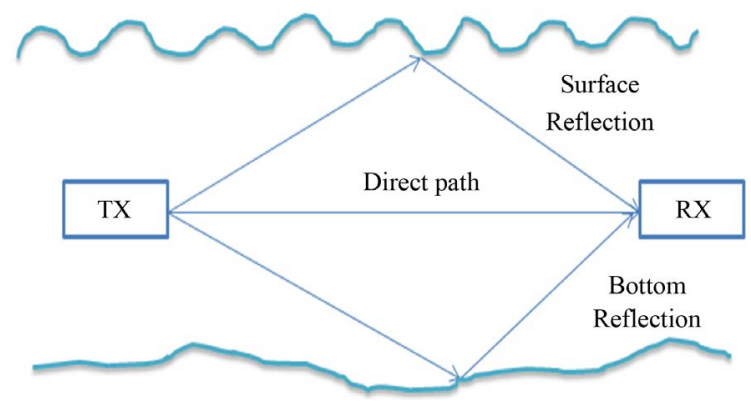

Figure 12. Shallow water multipath propagation: in addition to the direct path, the signal propagates via reflections from the surface and bottom. 
errors and due to this critical security packets can be lost. Wireless underwater communication channels can be snooped. Hackers and attackers may intercept the information transmitted and attempt to modify or drop data packets. Malicious attacks can create out-of-band connections via fast radio (above the water surface) and wired links. Since underwater communication sensors are mobile in nature so their relative distances vary with time.

\subsubsection{Jamming}

A jamming attack occurs by interfering with the physical channel by putting up carriers on the frequencies neighbor nodes use to communicate. Since underwater acoustic communication frequency bands are narrow so UWCNs are vulnerable to narrowband jamming.

\subsubsection{Worm Whole Attack}

A wormhole is an out-of-band connection created by the connection between two physical locations in a network with lower delay and higher bandwidth than ordinary connections. This connection uses fast radio or wired links to significantly decrease the propagation delay. In a wormhole attack the malicious node transfers some selected data packets received at one end of the wormhole to the other end using the out-of-band connection and re-injects them into the network. The effect is that false neighbor relationships are created because two nodes out of each other's range can erroneously conclude that they are in proximity of together due to the wormhole's presence.

\subsubsection{Sinkhole Attack}

In this attack a malicious node attempts to attract traffic from a particular area toward it. For example, the malicious node can announce a high-quality route.

\subsubsection{Hello Flood Attack}

In this attack a node receiving a HELLO packet from a malicious node may interpret that the adversary is a neighbor. This assumption is always false if the adversary uses high power for transmission.

\subsection{Security Requirements}

\subsubsection{Authentication}

Authentication in the network is the proof that the data was sent by a legitimate sender. It is very essential in military and safety-critical applications of UWCNs.

\subsubsection{Confidentiality}

This means that information is not accessible to unauthorized third party.

\subsubsection{Integrity}

It ensures that transmit information can't be change or alter by any adversary. Many underwater sensor applications such as water quality monitoring rely on the integrity of information.

\subsubsection{Availability}

The data should be available when needed by authorized user. Lack of availability due to denial-of-service attacks would especially affect time-critical aquatic exploration applications such as prediction of seaquakes.

\section{Conclusions}

In this topic we overviewed the main challenges for efficient communication in under water acoustic sensor wireless networks. We outlined the peculiarities of the underwater channel with particular reference to networking solutions. The ultimate objective of this topic is to encourage research efforts to lay down fundamental basics for the development of new advanced communication techniques for efficient under water communication and networking for enhanced ocean monitoring and exploration applications.

The aim of this paper is to build the acoustic communication and improve the underwater wireless communication channel by using IDMA scheme. 


\section{Future Enhancement}

IDMA used not only in cellular communication but also in under water communication. IDMA is fourth-generation (4G) research. Some more researches are under process for making the data transmission efficient. Open the new path for future optical communication with IDMA scheme incorporated with tree based interleavers. The Tree based interleavers can also be implemented with newly evolved diversity mechanism such as cooperative diversity with IDMA scheme.

\section{References}

[1] Berkhovskikh, L. and Lysanov, Y. (1982) Fundamentals of Ocean Acoustics. Springer, New York. http://dx.doi.org/10.1007/978-3-662-02342-6

[2] Quazi, A. and Konrad, W. (1982) Underwater Acoustic Communications. IEEE Communications Magazine, 24-29. http://dx.doi.org/10.1109/MCOM.1982.1090990

[3] Catipovic, J. (1990) Performance Limitations in Underwater Acoustic Telemetry. IEEE Journal of Oceanic Engineering, 15, 205-216. http://dx.doi.org/10.1109/48.107149

[4] Stojanovic, M. (1996) Recent Advances in High-Speed Underwater Acoustic Communications. IEEE Journal of Oceanic Engineering, 21, 125-136. http://dx.doi.org/10.1109/48.486787

[5] Essebbar, A., Loubet, G. and Vial, F. (1994) Underwater Acoustic Channel Simulations for Communication. Proceedings of OCEANS 94, Brest, 13-16 September 1994, 495-500.

[6] Falahati, A., Woodward, B. and Bateman, S. (1991) Underwater Acoustic Channel Models for 4800 b/s QPSK Signals. IEEE Journal of Oceanic Engineering, 16, 12-20. http://dx.doi.org/10.1109/48.64881

[7] Bjerrum-Niese, C., Bjorno, L., Pinto, M.A. and Quellec, B. (1996) A Simulation Tool for High Data-Rate Acoustic Communication in a Shallow-Water, Time-Varying Channel. IEEE Journal of Oceanic Engineering, 21, $143-149$. http://dx.doi.org/10.1109/48.486789

[8] Coatelan, S. and Glavieux, A. (1994) Design and Test of a Multicarrier Transmission System on the Shallow Water Acoustic Channel. Proceedings of OCEANS 94, Brest, 13-16 September 1994, 472. http://dx.doi.org/10.1109/OCEANS.1994.364244

[9] Stojanovic, M., Catipovic, J.A. and Proakis, J.G. (1994) Phase Coherent Digital Communications for Underwater Acoustic Channels. IEEE Journal of Oceanic Engineering, 19, 100-111. http://dx.doi.org/10.1109/48.289455

[10] Stojanovic, M., Catipovic, J.A. and Proakis, J.G. (1993) Adaptive Multichannel Combining and Equalization for Underwater Acoustic Communications. Journal of the Acoustical Society of America, 94, 1621-1631. http://dx.doi.org/10.1121/1.408135

[11] Stojanovic, M., Catipovic, J.A. and Proakis, J.G. (1995) Reduced-Complexity Multichannel Processing of Underwater Acoustic Communication Signals. Journal of the Acoustical Society of America, 98, 961-972. http://dx.doi.org/10.1121/1.413521

[12] Stojanovic, M., Proakis, J.G. and Catipovic, J.A. (1996) Performance of a High Rate Adaptive Equalizer on a Shallow Water Acoustic Channel. Journal of the Acoustical Society of America, 100, 2213-2219. http://dx.doi.org/10.1121/1.417930

[13] Ayela, G., Nicot, M. and Lurton, X. (1994) New Innovative Multi-Modulation Acoustic Communication System. Proceedings of OCEANS 94, Brest, 13-16 September 1994, 292-295.

[14] Shukla, M., Srivastava, V.K. and Tiwari, S. (2012) Analysis and Design of Tree Based Interleaver for Multiuser Receivers in IDMA Scheme. Proceedings of International Conference on Networks “ICON 2008”, Delhi, 1-4. 
Scientific Research Publishing (SCIRP) is one of the largest Open Access journal publishers. It is currently publishing more than 200 open access, online, peer-reviewed journals covering a wide range of academic disciplines. SCIRP serves the worldwide academic communities and contributes to the progress and application of science with its publication.

Other selected journals from SCIRP are listed as below. Submit your manuscript to us via either submit@scirp.org or Online Submission Portal.
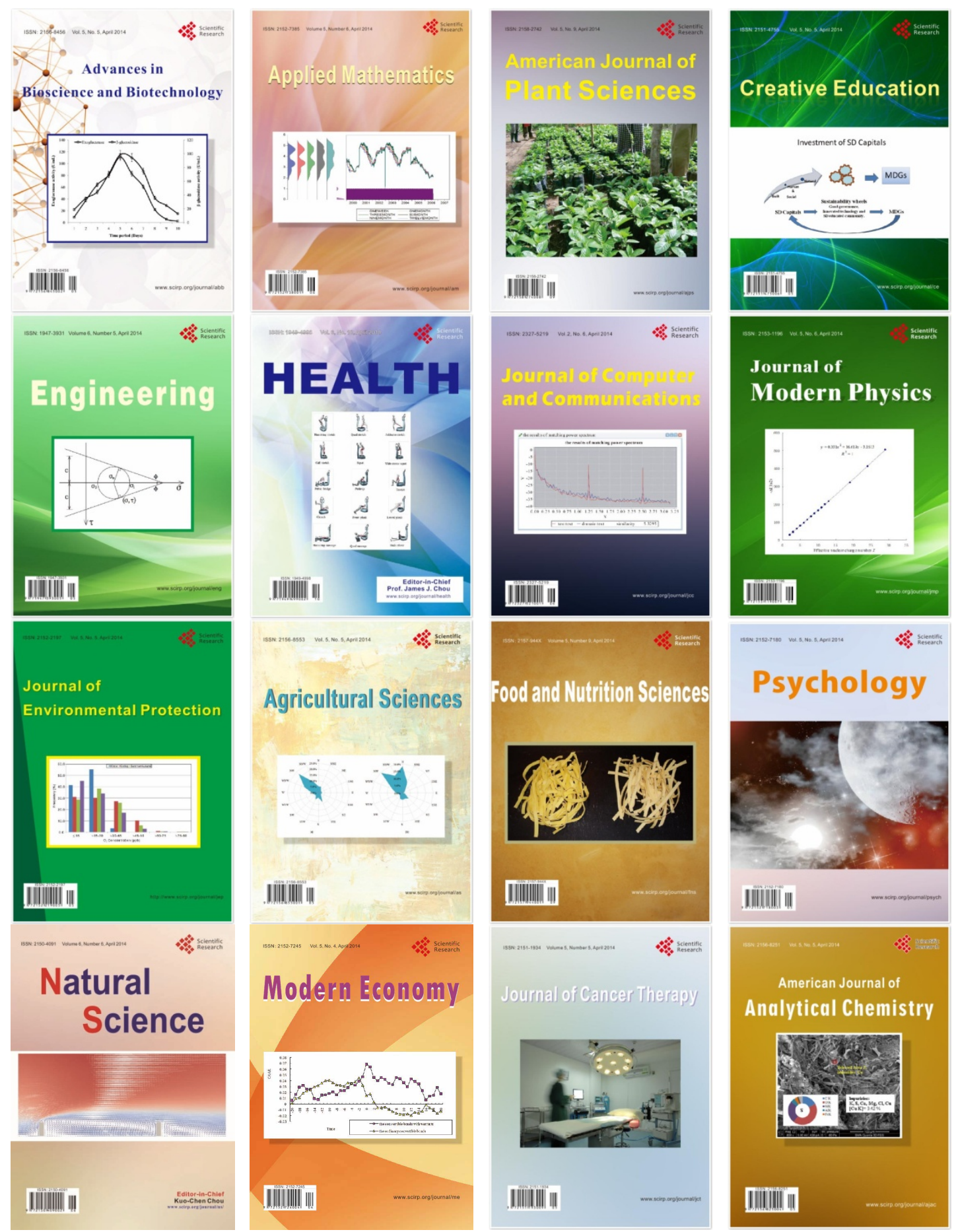